\title{
The role of pediatric hospitalists in coordinating the care provided to children with medical complexity
}

Over the past 30 years, the profile of patients requiring admission to children's hospitals has changed. Such change is the result of multiple factors, the most important of which include the advances accomplished in diagnostic methods, the development of new drugs and vaccines, minimally invasive surgeries, solid organ or hematopoietic stem cell transplantation, the survival rate of children with chronic diseases and technology-dependent, and evidence-based therapeutic decisions.

The frequency of acute infections, including bacterial pneumonia, arthritis, osteomyelitis, meningitis, exanthematous disease, has decreased, or if a child with an acute infection is hospitalized, the length of stay is shorter and the child usually completes treatment with oral antibiotics as an outpatient.

Viral respiratory tract infections are still prevalent among young children, but their hospitalization is reserved for those with hypoxemia and/or risk factors. A reduced oxygen saturation level accepted for oxygen therapy withdrawal and the generalized use of pulse oximeters have allowed to shorten hospitalization duration and reduce the risk for hospital-acquired infections.

With the enhancement of pre-surgical assessments, anesthesia selection, minimally invasive surgery, and an adequate pain management, most children may be hospitalized and discharged the same day of surgery or stay at the hospital for brief periods even after having undergone a major surgical procedure, which used to require several days in the hospital (e.g., closure of an atrial septal defect, splenectomy, cholecystectomy, nephrectomy, anti-reflux surgery, neuroendoscopic surgery for hydrocephalus, etc.).

Similarly, interventional hemodynamics allows patients to be discharged within 24 hours after having undergone closure of a patent ductus arteriosus or a ventricular septal defect, radiofrequency ablation for arrhythmias, or the treatment of aneurysms or other arteriovenous malformations.

It is not medically acceptable for a stable child with no social risks or life-threatening conditions to be hospitalized "for reaching a diagnosis." It is also not acceptable for a child and his/her family to travel hundreds of kilometers to see a doctor, with all the damages implied by estrangement, and the financial and family problems it carries, when a remote consultation and/or a blood or tissue sample submission for a specialized examination are an alternative.

However, hospital beds remain occupied by children with new emerging diseases or who survive thanks to technological and therapeutic advances.

The emergence of severe hospital- and community-acquired infections caused by multidrug resistant microorganisms is consuming significant resources across all levels of care.

Children with cancer, who even with the best therapeutic options may achieve a cure rate of $80 \%$, require frequent hospitalizations for treatment or because of concurrent conditions occurring in the months or years after diagnosis. Children who require solid organ or hematopoietic stem cell transplantation record the longest hospitalization rates, either before or after transplantation, usually as a result of concurrent or secondary diseases.

Technology-dependent children include those who have complex, chronic conditions involving several systems, such as encephalopathy, chronic pulmonary disease (including very low birth weight preterm infants), congenital anomalies, neuromuscular disease, intestinal insufficiency, immunodeficiencies, or autoimmune diseases.

What is the common denominator among these patients? How should children's hospitals transform themselves to provide more effective care to pediatric patients? How should limited human resources and available materials be managed?

This is not exclusively a local concern. Different countries and health systems have raised all these questions, although the answers require a thorough, specialized analysis. However, the first steps should have already been taken.

The common denominator of these patients is multisystem involvement and/or the fact that they require the assistance of diverse specialists 
and support services. The interaction among these specialists and support services will largely impact on the success of care. When multiple disciplines are not well managed and coordinated, care becomes fragmented and uses up resources inadequately (further tests, inadequate series, longer length of stay in the hospital, increased risk for drug interactions, etc.).

Interdisciplinary and team work appear as the best option. However, care should be focused on the patient and his/her needs, and every patient may have individual requirements. For care to be effective, team members should be included on a case-by-case basis. The participation of each team member should be coordinated, and team decisions should be adapted to the patient's situation in real time and on an ongoing basis.

At this point, the figure of the "pediatric hospitalist," as referred to in other countries, emerges as the most adequate health care provider to fulfill the role of care coordinator. The pediatric hospitalist is by the side and "on the side" of the patient and his/her family and, as Carlos Gianantonio, M.D. used to say, "... is the pivot around which the conceptual and practical convergence of multiple medical specialists revolves. The pediatric hospitalist is the patient's and the family's physician, manages and coordinates diagnostic and therapeutic procedures, and facilitates communication with other disciplines."

Not less important is the financial approach and resource optimization. Interdisciplinary care uses up a large number of highly specialized human resources. A smart management of care includes the acquisition of overall skills by pediatric hospitalists, the development of care guidelines that may be used in similar cases without the need to call all actors involved, the flexible incorporation of evidence-based advances, researching, and recording and distributing knowledge by creating a "collective memory" of each hospital's "best practices." The introduction of hospitalists to the role of care coordinators has been accompanied by a reduction in care costs, a lower number of unnecessary referrals to specialists, a shorter length of stay in the hospital, an improved quality of care, and greater satisfaction on part of patients and their families.

Actually, hospitalization is a limited time event in the life of these children and should be prevented or shortened as much as possible. The role of pediatric hospitalists or clinical pediatricians of children with medical complexity should not be limited to care during hospitalization. Their training should include every aspect necessary to participate in the longitudinal follow-up of these children and work in primary or secondary care facilities, close to the place of residence of patients and their families, where they could bring about a paradigm shift and make care provided to patients with medical complexity more humane.

Susana Rodríguez, M.D.

Hospital de Pediatría "Prof. Dr. Juan P. Garrahan”

http:/ /dx.doi.org/10.5546/aap.2017.eng.108

To cite: Rodríguez S. The role of pediatric hospitalists in coordinating the care provided to children with medical complexity. Arch Argent Pediatr 2017;115(2):108-109.

\section{REFERENCES}

1. Gianantonio CA. Comentarios sobre salud infantil y pediatría. Arch Arg Pediatr 1994;92(5):257-8.

2. Cohen E, Kuo DZ, Agrawal R, BerryJG, et al. Children with medical complexity: an emerging population for clinical and research initiatives. Pediatrics 2011;127(3):529-38.

3. Simon TD, MahantS, Cohen E. Pediatrichospital medicine and children with medical complexity: past, present, and future. Curr Probl Pediatr Adolesc Health Care 2012; 42(5):113-9.

4. Stucky ER, Maniscalco J, Ottolini MC, Agrawal R, et al. The Pediatric Hospital Medicine Core Competencies Supplement: a Framework for Curriculum Development by the Society of Hospital Medicine with acknowledgement to pediatric hospitalists from the American Academy of Pediatrics and the Academic Pediatric Association. J Hosp Med 2010;5 (Suppl 2):i-xv,1-114.

5. WachterRM,GoldmanL.Zeroto50,000.The20thAnniversary of the Hospitalist. New Engl J Med 2016;375(11):1009-11.

6. Lye PD, Committee on Hospital Care and Section on Hospital Medicine. Clinical report - Physicians' roles in coordinating care of hospitalized children. Pediatrics 2010;126(4):829-32. 